\title{
Genotyping of the Resistance Determinant of Neisseria Gonorrhoeae with Reduced Susceptibility to Ceftriaxone in Manaus-AM- Brazil
}

William Antunes Ferreira ${ }^{1}$, Waldemara De Souza Vasconcelos ${ }^{2}$ Jairo De Souza Gomes ${ }^{2}$ Maria De Fátima Pinto Da Silva ${ }^{2}$, Felipe Gomes Naveca ${ }^{3}$ and Cristina Motta Ferreira ${ }^{4}$

${ }^{1}$ PhD in Tropical and Infectious Disease, Fundação de Dermatologia Tropical e Venereologia Alfredo da Matta-FUAM, Brazil

${ }^{2}$ Pathology Technician, Fundação de Dermatologia Tropical e Venereologia Alfredo da Matta-FUAM, Brazil

${ }^{3} \mathrm{PhD}$ in Science (Microbiology), Instituto Leônidas e Maria Deane-FIOCRUZ-AM, Brazil

${ }^{4} \mathrm{PhD}$ in Tropical and Infectious Disease, 3Fundação de Hematologia e Hemoterapia do Amazonas -FHEMOAM, Brazil

Received: 18 May, 2017; Accepted: 15 June, 2017; Published: 28 June 2017

*Corresponding author: William Antunes Ferreira, PhD in Tropical and Infectious Disease, Fundação de Dermatologia Tropical e Venereologia Alfredo da Matta-FUAM, Manaus-Amazonas, Brazil. Tel: 55-9236325844; E-mail: wianfe@yahoo.com.br

\begin{abstract}
Gonorrhea is the second most prevalent sexually transmitted infection worldwide, with an estimated 78.3 million new cases. At the Alfredo da Matta foundation, gonorrhea as the main cause of urethral discharge with prevalence of $16.8 \%$. Gonococci have developed resistance to all the antibiotics leaving cephalosporins as the last option for treatment. In this report, we genotype the determinants of resistance to Extended Spectrum Cephalosporins, such as penA, ponA, porB, mtrR, pilQ, of a Neisseria gonorrhoeae strain, isolated from a male patient with urethral discharge. The ST1901was identified by MLST protocol and genotyping of the penA, showed mutations on regions F505L, A511V, A517G, N542H e P522S which confirmed the presence of gonococcus with reduced susceptibility to ceftriaxone in the region.
\end{abstract}

Keywords: Genotyping; MLST; N. Gonorrhoeae; Cephalosporin Resistance

\section{Text}

In last few decades, gonococci have developed resistance to all the antibiotics used as first line of treatment for gonococcal infections, leaving Extended- Spectrum Cephalosporins (ESCs) as the last remaining option for gonorrhea [1,2]. With reports of reduced susceptibility or resistance to ESCs from different regions, and due to therapeutic limitations, the infection has become a serious health problem to the point that disease complications can no longer be treated in the near future, besides the possibility of the gonococcus to evolve into "superbug" [3-7].

Gonorrhea is the second most prevalent sexually transmitted infection worldwide, with an estimated 78.3 million new cases in 2012 [8]. At the Alfredo da Matta foundation (Manaus-Brazil), gonorrhea appears as the main cause of urethral discharge, with an average of 513 cases in 20 years and prevalence of $16.8 \%$. In this report, we describe the molecular characteristics of the $N$. gonorrhoeae strain NgFUAM84, isolated from the urethral discharge of a male patient, with MIC of $0.064 \mu \mathrm{g} / \mathrm{mL}$ for ceftriaxone in E-test (AB Biodisk, Solna, Sweden) [9]. The determinants of resistance to ESCs: penA, ponA, porB, $m$ trR and pilQ were amplified by PCR (Proflex PCR System-Applied Biosystems) using primers previously described [10,11]. The sequencing of amplicons was performed on the ABI 3130 Genetic Analyzer (Applied Biosystems). The substitutions in the residues were analyzed using the software Geneious v.10.0.10 and identified by comparison with the sequences deposited in GenBank (Figure 1).

The molecular epidemiology was determined by Multi Locus Sequence Typing, performed according to the guidelines described in (http://pubmlst.org/neisseria). Clinical aspects, phenotypic characteristics, antimicrobial susceptibility test, analysis of the genes gyrA and parC, and identification of the ST225 for the NG-MAST (http://www.ng-mast.net)was performed as described earlier [9]. The NgFUAM84 was no betalactamase producer and were resistant to Ciprofloxacin (> $32 \mathrm{~g} /$ $\mathrm{mL})$, Chloramphenicol (3 g/mL), Ofloxacin $(>32 \mathrm{~g} / \mathrm{mL})$, reduced susceptibility to Penicillin $(0.75 \mathrm{~g} / \mathrm{mL})$ and Tetracycline $(0.75$ $\mathrm{g} / \mathrm{mL}$ ) [9]. The genotyping by MLST identified ST1901; clone associated with reduced sensibility and resistance to ECSs and is predominant worldwide [1].

Analysis of the gene ponA showed a single mutation at the L421P position, whereas in the gene $m t r R$, a single deletion of adenine (A) in the inverted position of the promoter region was identified. The NgFUAM84 also presented resistance determinant penB with substitutions at position G120K, A121D of loop 3 of PorB1b. These mutations have been associated with reduced sensitivity and resistance of gonococci to ECSs $[6,14]$. A single substitution on position G554D was identified in PilQ (Table 1). Analysis of the penA made possible identification of substitutions at positions F505L, A511V, A517G and P552S. The PBP2 protein showed similarity of $99.3 \%$ with the XXIV allele (GenBank 
accession number: FJ465093 and $99.7 \%$ and with strain NJ5 GenBank accession number: KF576657) [3,6]. Changes in the susceptibility of gonococcus to ceftriaxone were previously detected in Manaus however; genotyping of those strains was not performed. Recent studies (unpublished) show that gonococcus circulating in the region are still sensitive to the ESCs, however, elevated MICs to ceftriaxone has been detected [15]. Other authors have associated the presence of PBP2 mosaic alleles and mutations at A501V and A501T positions with reduced sensitivity and resistance to cefixime and ceftriaxone $[5,7,14]$. We did not identify these substitutions in NgFUAM84, however, the MIC of $0.064 \mu \mathrm{g} / \mathrm{mL}$ reinforces the possibility that non-mosaic penA mutations can increase the MICs of ESCs similar to those mediated by mosaic allele [3].

The mutations observed in the resistance determinants of ESCs in NgFUAM84 strain as well the identification of STs 225 and 1901, confirm the presence of gonococci with reduced susceptibility to ceftriaxone in the region and reinforces the need for monitoring of the susceptibility of gonococcus to these antibiotics and extensive research for better understanding of the resistance's mechanisms in order to maximize the effectiveness of ESCs in the treatment of gonorrhea. [4].

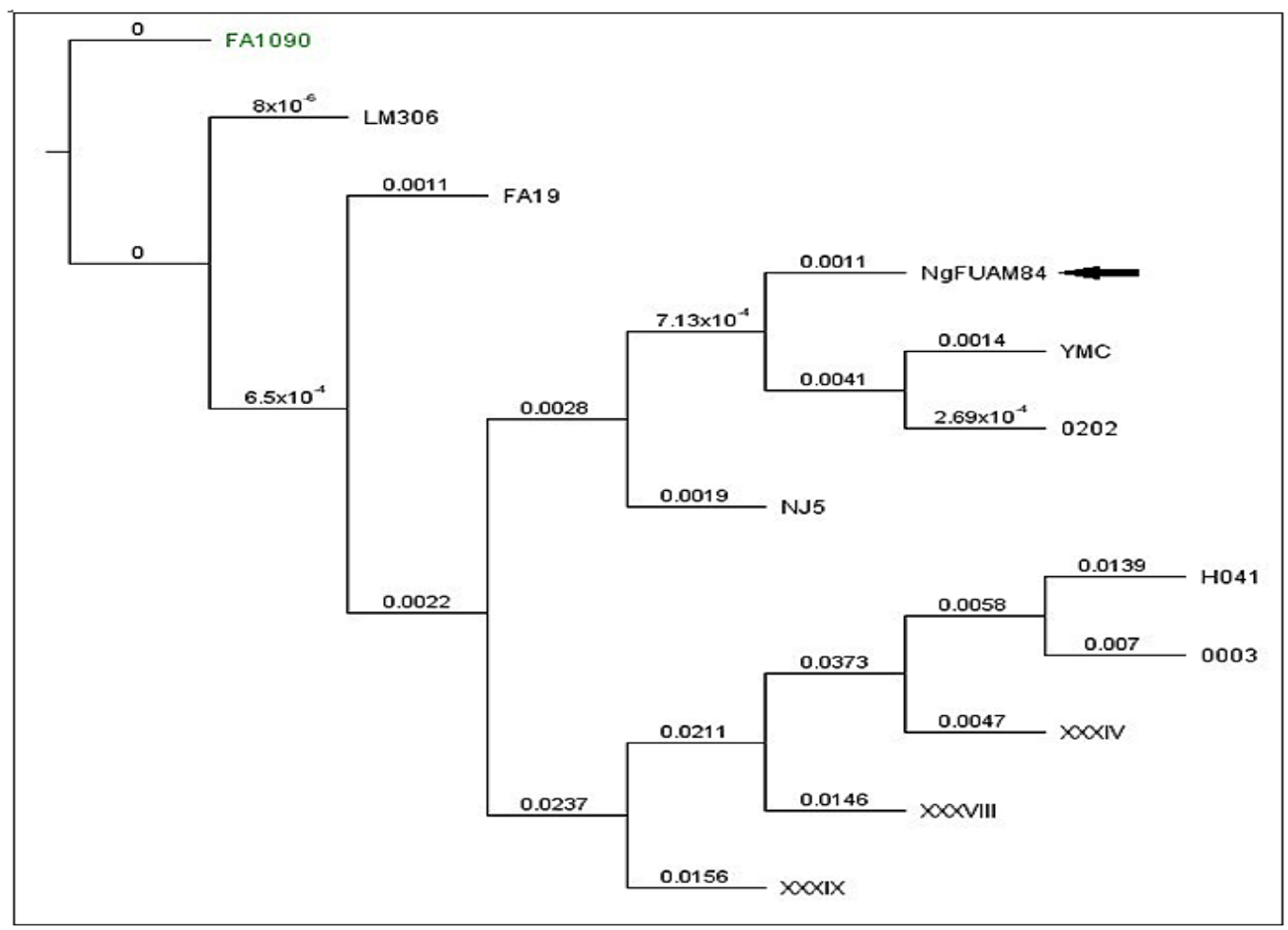

Figure 1:Cladogram of full-length of FUAM84's penA sequence with reference FA1090 (NC002946) and others: NgLM306 (NGOPENA-M32091.0) ; NgFA19; (NZ_AKCG00000000.1)WT ; NgYMC/NG02/37 (FJ465093.1) Lee ${ }^{3}$; Ng0202 (AB511946) Ohnishi13 ; NgNJ-5 (KF576657.1) Li ${ }^{6}$; NgH041 (AB546858) Ohnishi ${ }^{5}$; Ng0003 (AB511945) Ohnishi ${ }^{13}$; (HQ204552) NgXXXIV and (HQ204565) NgXXXVIII Allen ${ }^{11}$; (JF893455.1) NgXXXIX Martin unpublished. The arrow represents the rate of the sample of the study.

Table 1: Genotype characteristics - NgFUAM84

\begin{tabular}{|c|c|c|}
\hline Gene & Mutations & GenBank Access n $^{\circ}$ \\
\hline penA $\bullet$ & F505L, A511V, A517G and P552S & MF048800 \\
\hline ponA & L421P & MF062527 \\
\hline porB & G120K, A121D & MF048801 \\
\hline mtrR & Deleção A & MF095076 \\
\hline pilQ & G554D & MF095077 \\
\hline
\end{tabular}

-Presence of the extra codon on positions 346 of NgFUAM84 PBP2 


\section{Acknowledgment}

Prof. Dr. Ana Mika Dhyani, Júlio César Lima Sampaio and Victor Costa de Souza

\section{References}

1. Unemo M, Golparian D, Nicholas R, Ohnishi M, Gallay A, Sednaouie P. High-Level Cefixime and Ceftriaxone-Resistant Neisseria gonorrhoeae in France: Novel penA Mosaic Allele in a Successful International Clone Causes Treatment Failure. Antimicrob Agents Chemother. 2012;56(3):1273-1280. doi: 10.1128/AAC.05760-11

2. Nakayama S, Shimuta K, Furubayashi K, Kawahata T, Unemo M, Ohnishia M. New Ceftriaxone- and Multidrug-Resistant Neisseria gonorrhoeae Strain with a Novel Mosaic penA Gene Isolated in Japan. Antimicrob Agents Chemother. 2016;60(7):4339-4341. doi: 10.1128/ AAC.00504-16

3. Lee Sang G, Lee H, Jeong SH, Yong D, Chung GT, Lee YS, Chong Y, Lee K. Various penA mutations together with mtrR, porB and ponA mutations in Neisseria gonorrhoeae isolates with reduced susceptibility to cefixime or ceftriaxone. J Antimicrob Chemother. 2010;65(4):669675. doi: $10.1093 / \mathrm{jac} / \mathrm{dkp} 505$

4. Whiley DM, Limnios EA, Ray S, Sloots TP, Tapsall JW. Diversity of penA Alterations and Subtypes in Neisseria gonorrhoeae Strains from Sydney, Australia, that are Less Susceptible to Ceftriaxone. Antimicrob Agents Chemother. 2007;51(9):3111-3116.

5. Ohnishi M, Golparian D, Shimuta K, Saika T, Hoshina S, Iwasaku K, et al. Is Neisseria gonorrhoeae Initiating a future era of untreatable gonorrhea?: detailed characterization of the first strain with highlevel resistance to ceftriaxone. Antimicrob Agents Chemother. 2011;55(7):3538-3545. doi: 10.1128/AAC.00325-11

6. Li S, Su XH, Le WJ, Jiang FX, Wang BX, Rice PA. Antimicrobial susceptibility of Neisseria gonorrhoeae isolates from symptomatic men attending the Nanjing sexually transmitted diseases clinic (20112012): genetic characteristics of isolates with reduced sensitivity to ceftriaxone. BMC Infect Dis. 2014;14:622. doi: 10.1186/s12879-0140622-0
7. Gianecini R, Oviedo C, Stafforini G, Galarza P. Neisseria gonorrhoeae Resistant to Ceftriaxone and Cefixime, Argentina. Emerg Infect Dis. 2016; 22(6): 1139-1141.

8. World Health Organization. Report on Global sexually transmitted infection surveillance 2015:54. ISBN 978924156530 1. (NLM classification: WC140). Geneva. Switzerland.

9. William AF, Cristina MF, Felipe GN, Nayanne COSA, Waldemara SV, Jairo SG, et al. Genotyping of two Neisseria gonorrhoeae fluroquinoloneresistant strains in the Brazilian Amazon region. Mem Inst Oswaldo Cruz. 2011;106(5):629-631.

10. Tanaka M, Nakayama H, Huruya K, Konomi I, Irie S, Kanayama A, et al. Analysis of mutations within multiple genes associated with resistance in a clinical isolate of Neisseria gonorrhoeae with reduced ceftriaxone susceptibility that shows a multidrug-resistant phenotype. Int J Antimicrob Agents. 2006;27(1):20-26.

11. Allen VG, Farrell DJ, Rebbapragada A, Tan J, Tijet N, Perusini SJ, et al. Molecular Analysis of Antimicrobial Resistance Mechanisms in Neisseria gonorrhoeae isolates from Ontario, Canada. Antimicrob Agents Chemother. 2011;55(2):703-712. doi: 10.1128/AAC.00788-10

12. Liao M, Gu WM, Yang Y, Dillon JA. Analysis of mutations in multiple loci of Neisseria gonorrhoeae isolates reveals effects of PIB, PBP2 and MtrR on reduced susceptibility to ceftriaxone. J Antimicrob Chemother. 2011;66(5):1016-1023. doi: 10.1093/jac/dkr021

13. Ohnishi M, Watanabe Y, Ono E, Takahashi C, Oya H, Kuroki T, et al. Spread of a chromosomal cefixime-resistant penA gene among different Neisseria gonorrhoeae lineages. Antimicrob Agents Chemother. 2010;54(3):1060-1067. doi: 10.1128/AAC.01010-09

14. Whiley DM, Goire N, Lambert SB, Nissen MD, Sloots TP, Tapsall JW. Reduced susceptibility to ceftriaxone in Neisseria gonorrhoeae is spread internationally by genetically distinct gonococcal populations. J Antimicrob Chemother. 2011;66(5):1186-1187.

15. Ferreira WA, Vasconcelos WS, Pinto SMF, Gomes JS, Ferreira CM, Benzaken AS, et al. Resistência da a Antimicrobianos em Manaus: Período 2005-2006. DST - J bras Doenças Sex Transm. 2007;19(2):6569. 